\title{
Testing the Effectiveness of a Short-Term Stress Prevention Programme in Primary School Students
}

\author{
Chris Englert, Aline Bechler, Sarah Singh and Alex Bertrams
}

\begin{abstract}
The present study tested the effectiveness of a four-week, school-based, universal cognitive-behavioural stress prevention programme. The prevention programme included short daily exercises which were adopted from two well-validated anti stress trainings. The daily exercises took approximately 10-15 minutes on average and were performed during regular classroom sessions. Half of the classes were randomly assigned to the prevention group $(n=80)$, while the other half were sorted into the non-treatment control group, which did not take part in the stress prevention programme $(n=73)$. The students' physical and psychological stress-related symptoms were assessed five times (i.e., prior to the training and after each week of training). Their coping strategies, self-efficacy and self-control were also measured. It was hypothesized that in the prevention group students' physical and psychological stress-related symptoms would significantly decrease over time, compared to the non-treatment control group. Contrary to our predictions, the prevention programme did not lead to statistically significant changes in physical or psychological stress-related symptoms. The students' coping strategies, self-efficacy and self-control did not have an influence on the result patterns. The results indicate that short-term stress prevention programmes may not be as effective as long-term programmes.
\end{abstract}

Keywords: prevention; school; self-control; self-efficacy; stress

\section{Introduction}

Children are frequently confronted with challenges that can tax or exceed their psychological resources, which often elicits heightened levels of stress (Lazarus \& Folkman, 1984; Seiffge-Krenke, 2000). These stressors involve, amongst others, daily hassles, academic performance pressure or developmental tasks (de Anda et al., 2000; Donaldson, Prinstein, Danovsky, \& Spirito, 2000). The cross-national survey 'Health Behaviour in School-aged Children' (HSBC), which is regularly conducted by the World Health Organization (WHO), revealed that a high percentage of children aged 11-15 years report elevated stress levels (Inchley et al., 2016). For example, in the 2014 Swiss sample of the HSBC survey, over $22 \%$ of the 11 -yearold boys and over $17 \%$ of the 11-year-old girls reported moderate to high stress levels (Blaser \& Amstad, 2016).

Chronic stress exposure has been found to be associated with severe short- and long-term psychological (e.g., depression) and physical (e.g., cancer) problems (Cohen, Janicki-Deverts, \& Miller, 2007). Furthermore, there is growing evidence that chronic stress exposure during the early years of life can cause severe consequences for one's health across the lifespan (Dube et al., 2009; Matthews,

University of Bern, $\mathrm{CH}$

Corresponding author: Chris Englert

(christoph.englert@edu.unibe.ch)
2005). Considering the potential negative effects of stress on health and wellbeing, it seems very important to support children in developing their abilities to efficiently cope with stressful situations (McNamara, 2000). Given the fact that coping strategies are primarily developed during adolescence, focusing on opportunities to improve coping skills during adolescence is highly important (Currie, Hurrelmann, Settertobulte, Smith, \& Todd 2000). Several prevention programmes have been developed and evaluated in previous years, indicating that strategies for improving children's coping capacities are highly important (Grant et al., 2003); however, most of these programmes are performed outside the classroom, as the exercises are too long and may interfere with regular classroom sessions (Hampel \& Petermann, 2003). This limitation may lead to a high number of dropouts and influence the effectiveness of the respective programme. To ensure that most students take part in a stress prevention programme, it seems beneficial to conduct the respective exercises during regular classroom sessions, which is why shorter exercises should be developed (Lohaus, 2010).

The goal of the present study was to develop and evaluate a four-week stress prevention programme that includes short daily exercises, which can be easily completed during regular classroom sessions under the supervision of a teacher. The exercises were sourced from two well-validated cognitive-behavioural stress prevention programmes: the Anti-Stress Training for children (AST) 
(Hampel \& Petermann, 2003) and the Stress Prevention Training for primary school children (Klein-Heßling \& Lohaus, 2000). Both of these two programmes contain cognitive-behavioural techniques (e.g., cognitive restructuring and problem solving) aimed at helping students to develop emotion-focused coping skills to reduce the actual sensations of psychological stress (e.g., relaxation techniques) and to establish problem-focused coping skills so that they can effectively deal with daily stressors in the future (see Figure 1 for our logical model). The daily exercises we included in the present study took approximately 10-15 minutes on average and were performed during regular classroom sessions led by the respective teachers. This longitudinal study tested the core hypothesis, that the students' physical and psychological stress-related symptoms would significantly decrease while participating in the four-week stress prevention programme, compared to a non-treatment control group which did not take part in the stress prevention programme.

\section{Methods \\ Participants}

An a-priori power analysis was conducted using $\mathrm{G}^{*}$ Power, revealing that we needed a sample of $N=141$ students to detect at least a medium effect (parameters: $\mathrm{f}=.30$, $\alpha=.05,1-\beta=.85$ ) (Faul, Erdfelder, Lang, \& Buchner, 2007). We randomly contacted several schools in Switzerland via telephone and asked them if they were willing to participate in the present study (please note that the protocols for recruitment and communication were deleted after completion of the study and therefore cannot be included). A total of 153 third- and fourth-grade students $\left(M_{\text {age }}=9.50, S D_{\text {age }}=0.62 ; 78\right.$ females $)$ from 10 classes in 4 Swiss schools volunteered to participate. We had to exclude an additional 13 students, either because language difficulties precluded following instructions or because they did not appear at more than one time of measurement.

This study has been approved by the local ethics committee of the faculty of Human Sciences at the University of Bern, Switzerland (see Ethical_Approval in the Supplemental Material available online). All procedures performed in this study were in accordance with the ethical standards of the institutional and/or national research committee and with the 1964 Helsinki declaration and its later amendments. We obtained written informed consent from the parents and the students prior to their inclusion in the study. The data collection took place between January and March 2017.

\section{Procedure}

Five classes from two schools were randomly assigned to the prevention group $(n=80)$, while five classes from the remaining two schools were sorted into the non-treatment control group, which did not take part in the stress prevention programme $(n=73)$. The randomization procedure was conducted via random.org. The study lasted four weeks and the students in both groups were tested at five times of measurement: prior to starting the programme (T1) and on the Friday of each week the programme was conducted (T2-5). A follow-up assessment could not be implemented due to organisational difficulties and time restrictions among the respective schools. To match the data with each student, all students generated a unique, anonymous code by writing down the first letter of their father's name, the first letter of their mother's name, and the date of the day they were born. For each questionnaire administered, overall scores were generated by averaging each participant's answers given in the respective questionnaire; higher scores are indicative of a higher value for the corresponding variable.

\section{Operationalisations}

Baseline assessment. At the first time of measurement, the students generated their anonymous codes and reported their demographic information (i.e., age, gender and native language; see Questionnaire_Baseline in the Supplemental Material available online for the full baseline questionnaire). To assess habitual coping tendencies, the students then worked on the 'German Coping Questionnaire for Children and Adolescents' (Stressverarbeitungsfragebogen für Kinder und Jugendliche; SVF-KJ; Hampel \& Petermann, 2016). The SVF-KJ requests students to rate a series of 36 possible coping responses to interpersonal stressors and 36 possible coping responses to academic stressors. Each response has to be rated on a scale of 1 ('not at all') to 5 ('all the time'), indicating how likely they would apply the coping response in the respective situation. Considering that the present study examined academic stress, the subscale for interpersonal stress was omitted, a decision that has been made in previous research as well (e.g., Hampel, Meier, \& Kümmel, 2008). The scale for academic stress assessed nine coping strategies, each represented by four items, including: minimisation (e.g., 'I say to myself: "It is

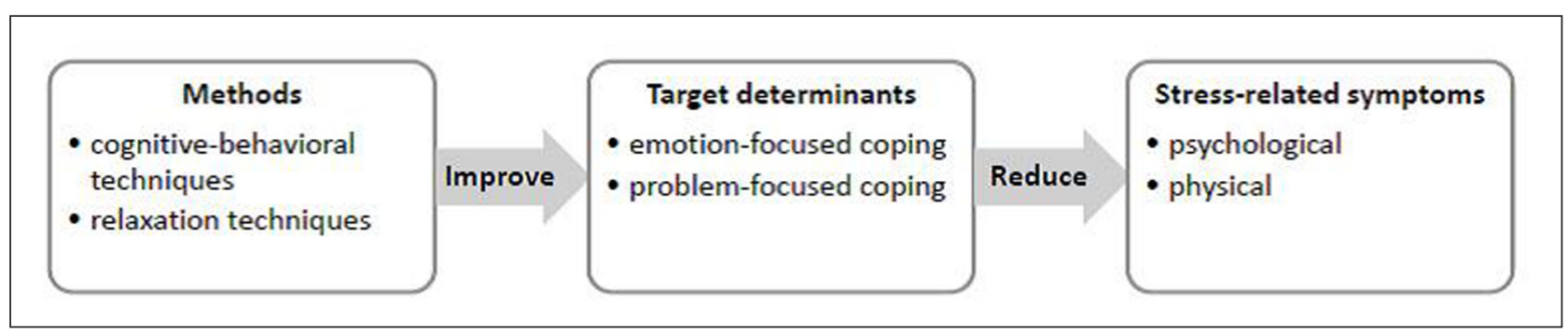

Figure 1: Logical model of the present study: It is assumed that practicing cognitive-behavioural techniques and relaxation exercises should improve emotion-focused as well as problem-focused coping skills, which in turn should reduce physical as well psychological stress-related symptoms. 
not that important".'), distraction/recreation (e.g., 'I just do something that I enjoy.'), situation control (e.g., 'I do something about it.'), positive self-instruction (e.g., 'I say to myself: "I can solve this problem".'), social support (e.g., 'I talk to someone about the problem.'), passive avoidance (e.g., 'I like to pretend I am sick.'), rumination (e.g., 'I worry about the situation the whole time.'), resignation (e.g., 'I say to myself: "I cannot do anything about it".') and aggression (e.g., 'I get a bad temper.').

Previous research has shown that self-efficacy and selfcontrol are important internal resources that can act as preventative measures against stress (e.g., Hampel et al., 2008); as such, the participants of the present study completed the 'Resource Questionnaire for Children and Adolescents' (Fragebogen zu Ressourcen im Kindesund Jugendalter; FRKJ 8-16; Lohaus \& Nussbeck, 2016). Self-efficacy (e.g., 'If I set a goal for myself, I will reach it.') and self-control (e.g., 'I am good at focusing on a given task.') were measured with six items each, which were rated on four-point Likert scales (i.e., 1 'never' to 4 'always').

Weekly assessments. To measure stress-related symptoms at each time point (T1-5), we administered the symptom checklist of the revised 'German Stress and Coping Questionnaire for Children and Adolescents' (Fragebogen zur Erhebung von Stress und Stressbewältigung im Kindes- und Jugendalter; SSKJ 3-8; Lohaus, Eschenbeck, Kohlmann, \& Klein-Heßling, 2006). The students were asked to report how often they experienced six physical stress-related symptoms (e.g., 'How often have you experienced dizziness?') and twelve psychological stress-related symptoms (e.g., 'How often have you been sad?') during the week (i.e., 1 'never', 2 'once', 3 'more than once'). The SSKJ 3-8 was first filled out at T1 (physical stress-related symptoms: prevention group, $M=1.45, S D=0.37$; non-treatment control group, $M=1.63, S D=0.34 ; \alpha=.49$. Psychological stress-related symptoms: prevention group, $M=1.72, S D=0.41$; nontreatment control group, $M=1.60, S D=0.45 ; \alpha=.78$ ). The SSKJ 3-8 was also administered to both groups on every Friday during the four weeks of the programme (for descriptive statistics, see Table 1). The students' stress-related symptoms were assessed five times in total for both groups (see Questionnaire_Weekly in the Supplemental Material available online for the full weekly questionnaire).

Stress prevention programme. The stress prevention programme lasted four weeks and included exercises adapted from two well-validated cognitive-behavioural stress prevention programmes: AST (Hampel \& Petermann, 2003) and Stress Prevention Training (Klein-Heßling \& Lohaus, 2000). Both source programmes are based on the concept of stress proposed by Lazarus and Folkman (1984), as well as Meichenbaum's (1985) 'Stress Inoculation Training'. We chose exercises that took, on average, less than 15 minutes to complete, as the goal of the present study was to develop a short stress prevention programme that could be easily integrated into regular classroom sessions. The programme of the present study was highly structured; students in the prevention group received a manual that included all exercises and instructions for every school day during the four weeks, which were conducted during regular classroom lessons (see Manual_Children in the Supplemental Material available online for the manual).

The first session of the programme was led by one of the researchers of the present study, while the remaining

Table 1: Descriptive Statistics: Means and Standard Deviations for the Control Measures.

\begin{tabular}{lccccc} 
& & \multicolumn{4}{c}{ Experimental Group } \\
\cline { 3 - 6 } Variables & & \multicolumn{1}{c}{ Prevention } & \multicolumn{2}{c}{ Control } \\
\cline { 4 - 6 } & .58 & 3.09 & 0.69 & 3.18 & 0.81 \\
\hline SVF-KJ minimisation & .67 & 3.47 & 0.84 & 3.48 & 0.89 \\
SVF-KJ distraction/recreation & .71 & 3.79 & 0.80 & 3.60 & 0.84 \\
SVF-KJ situation control & .70 & 3.75 & 0.71 & 3.62 & 0.87 \\
SVF-KJ positive self-instructions & .70 & 3.42 & 0.77 & 3.41 & 0.85 \\
SVF-KJ social support & .53 & 2.52 & 0.82 & 2.71 & 0.85 \\
SVF-KJ passive avoidance & .73 & 3.05 & 0.90 & 2.96 & 0.89 \\
SVF-KJ rumination & .57 & 2.29 & 0.73 & 2.31 & 0.73 \\
SVF-KJ resignation & .60 & 2.37 & 0.76 & 2.49 & 0.81 \\
SVF-KJ aggression & .83 & 2.80 & 0.58 & 2.59 & 0.46 \\
FRKJ 8-16 self-efficacy & .66 & 2.90 & 0.51 & 2.82 & 0.53 \\
FRKJ 8-16 self-control & &
\end{tabular}

Note. $n=80$ in the prevention group, $n=73$ in the non-treatment control group. Overall scores of a psychometric scale were obtained by averaging the responses to the scale items. SVF-KJ refers to the Stressverarbeitungsfragebogen für Kinder und Jugendliche (German Coping Questionnaire for Children and Adolescents; response scale ranging from 1 [not at all] to 5 [all the time]); FRKJ 8-16 refers to the Fragebogen zu Ressourcen im Kindes- und Jugendalter (Resource Questionnaire for Children and Adolescents; response scale ranging from 1 [never] to 5 [always]). 
sessions were led by the respective classroom teachers, who received a thorough introduction to the concept of stress and all exercises depicted in the manual by the researcher before the programme started. The researcher also handed out a four-week schedule to the teachers, showing which exercises to perform on which day (see Timetable in the Supplemental Material available online for the four-week schedule). Each session started with a short audio-relaxation technique (cf. McCallie, Blum, $\&$ Hood, 2006), followed by the actual exercise for the given day. At the end of each week, the students reported their stress-related symptoms on the SSKJ 3-8 checklist (Lohaus et al., 2006). Each Monday, the students wrote down their tasks and responsibilities for each day of the upcoming week; previous research has demonstrated that thorough planning can be a helpful strategy for reducing stress-related symptoms (e.g., Misra \& McKean, 2000).

On the first day of the programme, the researcher explained the concept of stress in detail (cf. Lazarus \& Folkman, 1984), demonstrated how to use the training manual and taught the students how to perform the relaxation techniques. The daily sessions over the following four weeks were led by the respective classroom teachers. The goal for the first week was to increase the students' knowledge and understanding of stress by asking them to brainstorm what they knew about the concept of stress and how they coped with stressful experiences, and to write down situations in which they were proud of themselves for handling a stressful episode. During the next three weeks, the students performed daily exercises designed to a) improve their understanding of the relations between their thoughts and stress and to b) develop a more positive self-evaluation.

On the final day of the programme, the students were thanked, debriefed and received a small gift.

\section{Results}

\section{Analysis plan}

We decided a-priori to allow two missing values for each questionnaire. We also inspected all questionnaires for whether the students completed them seriously or not. In case the students did not complete them seriously, we had to exclude these students from our analyses. However, this was not the case in our study.

To analyse whether the prevention and the nontreatment control group differed in their habitual use of certain coping strategies (as measured by the SVF-KJ; Hampel \& Petermann, 2016), in their self-control resources, or in their self-efficacy (as measured by the FRKJ 8-16; Lohaus \& Nussbeck, 2016), between-subjects analyses of variance (ANOVA) were conducted.

A mixed $2 \times 4$ between-/within-subjects analysis of covariance (ANCOVA) was conducted to test the effect of the stress prevention programme on physical stressrelated symptoms. The baseline scores for physical stressrelated symptoms were added as a covariate (for details on this procedure, see Lohaus, Fridrici, \& Maass, 2009). The experimental group (i.e., prevention vs. non-treatment control group) was added as the between-subjects factor, time of measurement (T2-5) as the within-subjects factor, and the physical stress-related symptoms were added as the dependent variables. In the same vein, to test whether the stress prevention programme influenced psychological stress-related symptoms, we conducted a mixed $2 \times 4$ between (i.e., prevention group vs. non-treatment control group) - within-subjects (i.e., time of measurement [T25]) ANCOVA. The baseline scores for psychological stressrelated symptoms were added as a covariate.

\section{Preliminary analyses}

Descriptive statistics resulting from the preliminary analyses are illustrated in Table 1. The full data set is available at https://figshare.com/articles/Untitled_Item/6264767. An ANOVA revealed that the prevention and the nontreatment control group did not differ significantly in any of the nine SVF-KJ scales (Hampel \& Petermann, 2016), indicating that there were no differences concerning the habitual use of certain coping strategies ( $p s>.142$ ).

Furthermore, between-subjects ANOVAs demonstrated that the two groups did not differ significantly in their selfcontrol resources, $F(1,151)=0.73, p=.396, \eta_{p}^{2}=.01$; however, participants from the prevention group displayed significantly higher self-efficacy scores than participants from the non-treatment control group, $F(1,151)=5.93$, $p=.016, \eta_{p}^{2}=.04$. Therefore, self-efficacy was added as a covariate to the main analyses.

\section{Main analyses}

As previously mentioned, we conducted a mixed $2 \times 4$ between (i.e., prevention vs. non-treatment control group)/within (time of measurement)-subjects analysis of covariance (ANCOVA) to test the effect of the stress prevention programme on physical stress-related symptoms (for descriptive statistics, see Table 2), while adding the baseline scores for physical stress-related symptoms as a covariate. We also added self-efficacy as a second covariate, as the preliminary analysis revealed significant differences in self-efficacy between the two groups (please note that the following results did not change significantly when excluding self-efficacy as a covariate). There was neither a significant main effect of time of measurement, $F(3,146)$ $=0.38, p=.766, \eta_{p}^{2}=.01$, nor of the experimental group (i.e., prevention vs. non-treatment control group) on physical stress-related symptoms, $F(1,148)=0.10, p=.754$, $\eta_{p}^{2}=.00$. The interaction between the two also did not reach statistical significance, $F(3,146)=0.26, p=.851$, $\eta_{p}^{2}=.00$, indicating that the prevention programme did not have a significant effect on relieving physical stressrelated symptoms (see Figure $\mathbf{2}$ for a visualisation of the effects of the stress prevention programme on physical stress-related symptoms).

Contrary to our predictions, we also found no effect of time measurement, $F(3,142)=1.17, p=.322, \eta_{p}^{2}=.02$, no effect of the experimental group (i.e., prevention vs. non-treatment control group), $F(1,144)=2.32$, $p=.130, \eta^{2}=.02$, and no effect of their interactions on psychological stress-related symptoms, $F(3,142)=$ $1.00, p=.394, \eta_{p}^{2}=.02$ (see Figure 3 for a visualisation of the effects of the stress prevention programme on psychological stress-related symptoms). 
Table 2: Descriptive Statistics: Means and Standard Errors for the Main Measures.

\begin{tabular}{|c|c|c|c|c|c|}
\hline \multirow[t]{3}{*}{ Variables } & \multirow[b]{3}{*}{$\alpha$} & \multicolumn{4}{|c|}{ Experimental Group } \\
\hline & & \multicolumn{2}{|c|}{ Prevention } & \multicolumn{2}{|c|}{ Control } \\
\hline & & $M$ & $S E$ & $M$ & $S E$ \\
\hline T2 SSKJ 3-8 psychological ${ }^{\mathrm{a}, \mathrm{b}}$ & .84 & 1.68 & 0.04 & 1.72 & 0.04 \\
\hline T3 SSKJ 3-8 psychologicala,b & .85 & 1.55 & 0.04 & 1.66 & 0.04 \\
\hline T4 SSKJ 3-8 psychologicala,b & .87 & 1.56 & 0.05 & 1.61 & 0.05 \\
\hline T5 SSKJ 3-8 psychologicala, & .85 & 1.48 & 0.04 & 1.56 & 0.04 \\
\hline T2 SSKJ 3-8 physicala,c & .71 & 1.52 & 0.04 & 1.53 & 0.05 \\
\hline T3 SSKJ 3-8 physical ${ }^{\mathrm{a}, \mathrm{c}}$ & .68 & 1.46 & 0.04 & 1.46 & 0.05 \\
\hline T4 SSKJ 3-8 physicala,c & .78 & 1.49 & 0.05 & 1.53 & 0.05 \\
\hline T5 SSKJ 3-8 physicala,c & .74 & 1.41 & 0.04 & 1.41 & 0.05 \\
\hline
\end{tabular}

Note. $n=80$ in the prevention group, $n=73$ in the non-treatment control group. Overall scores of a psychometric scale were obtained by averaging the responses to the scale items. SSKJ 3-8 refers to the Fragebogen zur Erhebung von Stress und Stressbewältigung im Kindes- und Jugendalter (response scale: $1=$ never, $2=$ once, $3=$ more than once). T2: Friday of the first week; T3: Friday of the second week; T4: Friday of the third week; T5: Friday of the fourth week. ${ }^{a}$ self-efficacy added as covariate. ${ }^{b}$ baseline stress-related psychological symptoms added as covariate. ${ }^{\mathrm{c}}$ baseline stress-related physical symptoms added as covariate.

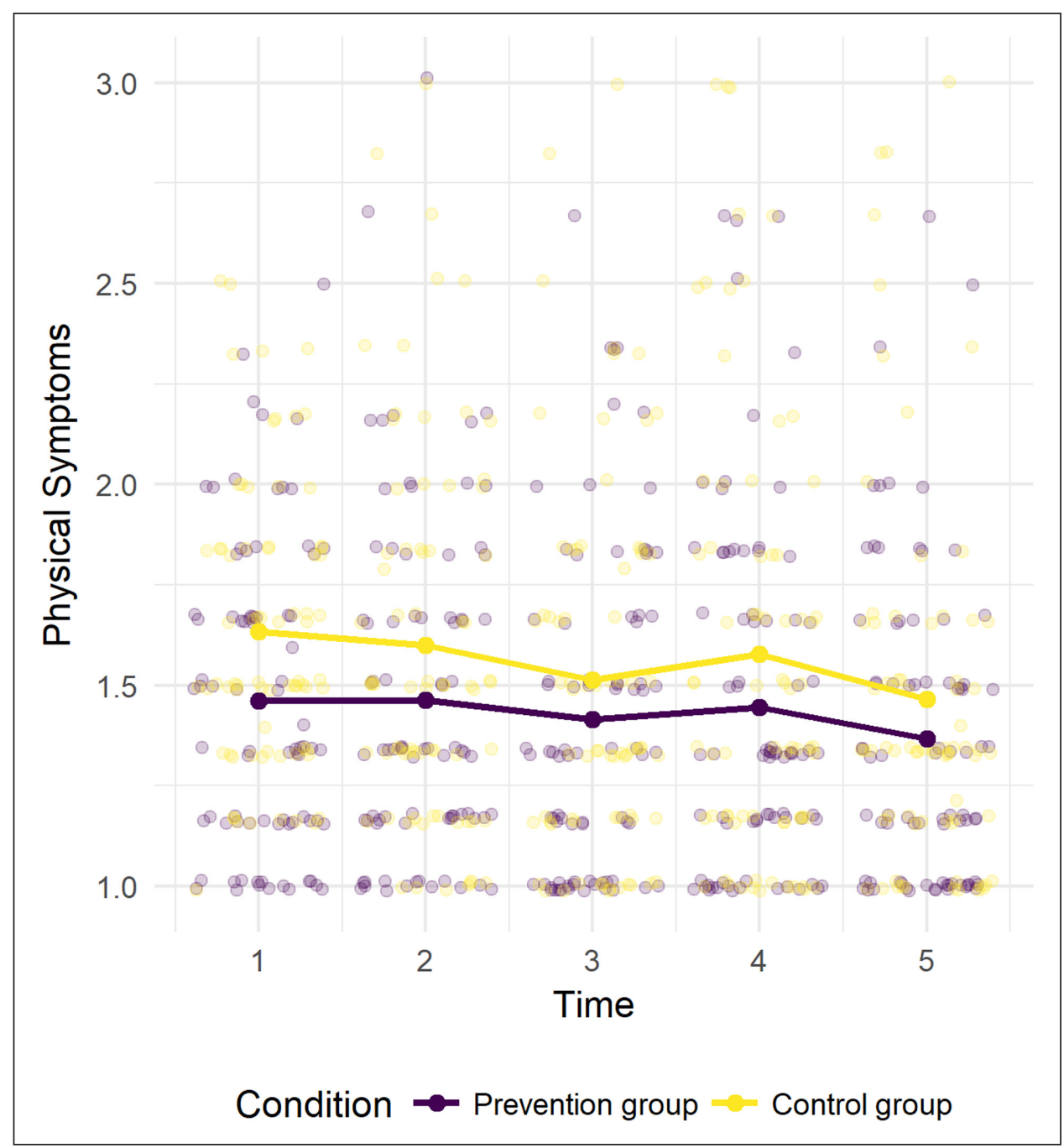

Figure 2: Visualisation of the effects of the stress prevention programme on physical stress-related symptoms (X-axis = Time of measurement; Y-axis = Aggregated physical stress-related symptoms; purple line = prevention group; yellow line = non-treatment control group; dots = Physical stress-related symptoms for each participant at each time of measurement). 


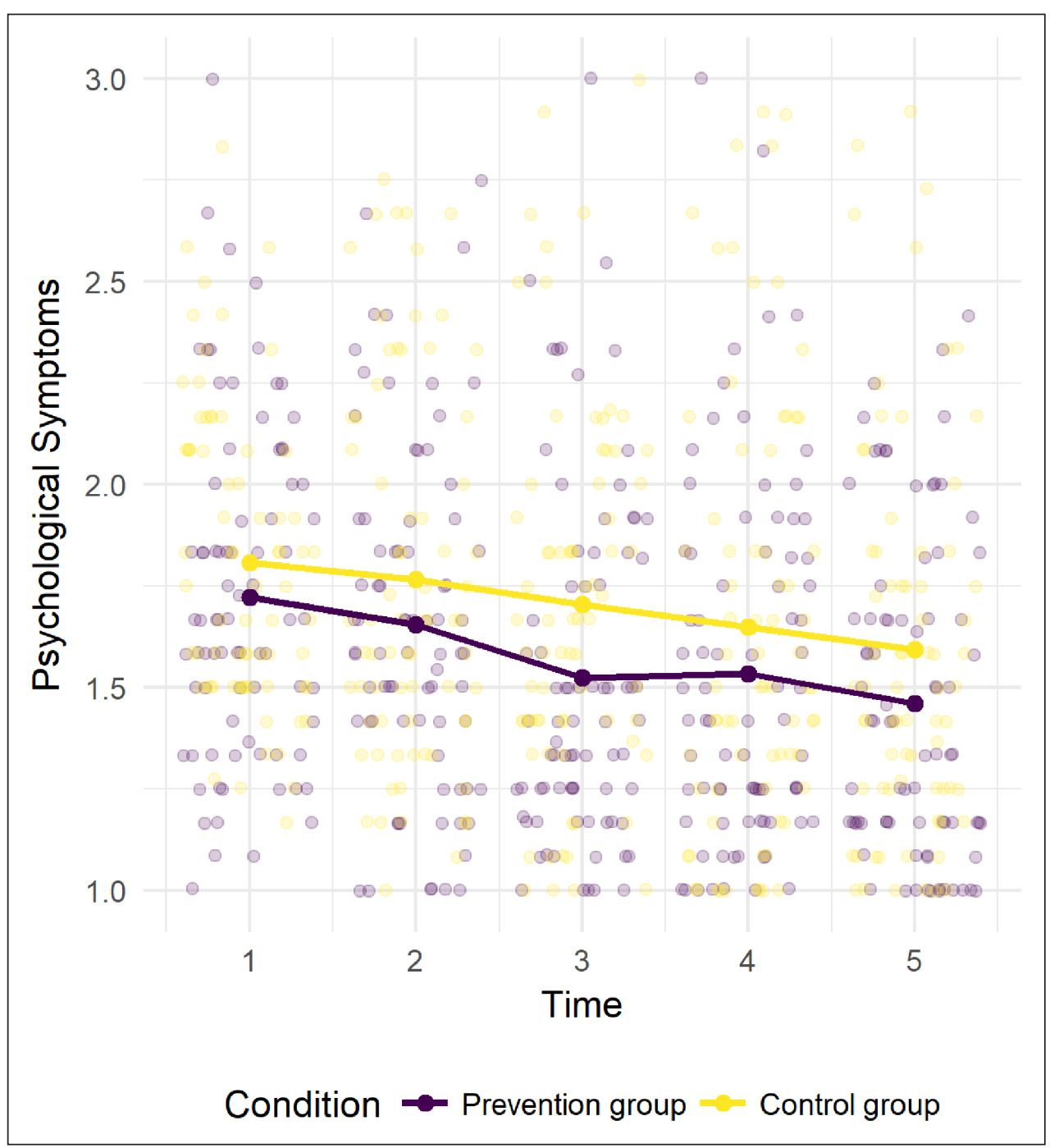

Figure 3: Visualisation of the effects of the stress prevention programme on psychological stress-related symptoms (X-axis = Time of measurement; Y-axis = Aggregated psychological stress-related symptoms; purple line = prevention group; yellow line = non-treatment control group; dots = Psychological stress-related symptoms for each participant at each time of measurement).

\section{Discussion}

Chronic stress is related to a wide variety of psychological and physical problems (Cohen et al., 2007), which often translates from adolescence into adulthood (Dube et al., 2009; Matthews, 2005). Several researchers have pointed out the necessity of developing stress prevention programmes for children and implementing these programmes into their daily lives at school (e.g., Lohaus, 2010). The present study investigated the effectiveness of a four-week universal stress prevention programme, which included exercises from two well-validated anti-stress training programmes (i.e., Hampel \& Petermann, 2003; Klein-Heßling \& Lohaus, 2000). The daily exercises were performed during regular classroom sessions, lasting approximately 10-15 minutes on average. We chose exercises that were relatively short and easy to integrate into regular lessons. We chose this approach because we did not want to interfere with the teacher's tuition and also wanted to minimise programme dropout rates (cf. Lohaus, 2010).
While the students expressed satisfaction with the prevention programme and participated in most of the exercises, the results were contrary to our expectations. During the four-week period, psychological and physical stress-related symptoms decreased in both groups; however, we did neither find a statistically significant effect of time of measurement nor of our prevention programme overall. One reason may be that the exercises were simply too short to change the students' pre-existing coping habits. Even though the primary goal of the present study was to develop a short-term stress prevention programme that could be easily integrated into regular classroom sessions, it seems it is more beneficial to implement prevention programmes including exercises of longer durations (see de Anda, 1998).

\section{Limitations}

An important limitation lies in the fact that we did not include any follow-up assessments after the programme ended. It is possible that the programme has an effect 
only after a certain period, which is why future studies should consider additional measurements. By doing so, it would be possible to determine the potential long-term effects of the training.

Furthermore, we exclusively focused on children, even though previous research has outlined that parents play a vital role in their children's stress experiences (e.g., Hampel et al., 2008). For example, the Anti-Stress Training includes information sessions in which parents receive information on the concept of stress, coping strategies and the key components of the stress prevention programme (see Hampel \& Petermann, 2003). The idea behind this holistic approach is that parents can help their children implement novel coping strategies, also serving as role models for coping with stress (e.g., Power, 2004).

We would also like to mention that the internal consistencies were not satisfactory for all applied measures in the current study. At T1, the $\alpha$-coefficient for the SSKJ 3-8 measuring physical stress-related symptoms was rather low $(\alpha=.49)$. However, previous research has delivered sound empirical evidence for the reliability and the validity of all the scales we administered (Eschenbeck, Kohlmann, Lohaus, \& Klein-Heßling, 2006). Furthermore, at the other times of measurement the internal consistencies for the SSKJ 3-8 were all satisfactory.

\section{Potential explanations}

One potential explanation for the non-significant findings in the current study might be that the teachers were not fully committed to the programme and did not make sure that the exercises were exactly performed as originally intended. We tried to minimize this risk by giving all teachers a proper introduction to the concept of stress before the programme started, by handing out a fourweek schedule which explained the training content for each day in detail, and by depicting all exercises in the training manual. Furthermore, the exercises were highly standardized (e.g., audio files for the relaxation units; training manual). We asked each teacher to register if the exercises had been conducted as planned for each day and the feedback we received was consistently positive.

In the same vein, it could be argued that the students' compliance to participate in the training was rather low. While we did not explicitly measure compliance, we did assess whether the students had worked on the daily exercises depicted in the manual, which was mostly the case in the present study. We also received verbal feedback from the students which was consistently positive.

It might also be possible that the training, while not immediately affecting the stress-related symptoms, did have a positive effect on students' coping skills. However, we only measured habitual coping tendencies at baseline, which is why future studies should consider to also measure coping skills after the four-week training.

\section{Recommendations for future research}

Lohaus (2010) stresses that students' motivation toward participating in stress prevention programmes seems rather weak, and dropouts are a serious problem. He recommended including internet-based e-learning tools in stress prevention programmes, because children and adolescents are often interested in innovative multimedia tools (see also Vandewater et al., 2007). The advantage of using online tools is that they can be easily accessed and learners can decide for themselves how and when they want to access them (Barak, Klein, \& Proudfoot, 2009; Taylor, Jobson, Winzelberg, \& Abascal, 2002). Internetbased programmes have been developed and validated for treating several physical and psychological problems (e.g., depression; Richards \& Richardson, 2012). In the same vein, a recent study by Lohaus (2010) suggests that e-learning tools may help maximise the effects of traditional stress prevention programmes (see also Lohaus et al., 2009).

Given that children and adolescents are often less motivated toward participating in long-term stress prevention programmes, future research should focus on developing more effective short-term stress prevention programmes, potentially including e-learning elements (Lohaus, 2010). However, given the fact that we did not find a statistically significant effect of our short-term prevention program on stress-related symptoms, we recommend that researchers and practitioners developing short-term prevention programs in the future ensure these undergo careful development and testing prior to implementation.

\section{Data Availability Statement}

Full data set available at https://figshare.com/articles/ Untitled_Item/6264767.

\section{Additional Files}

The additional files for this article can be found as follows:

- S File 1. Tier 1 manuscript submission. DOI: https:// doi.org/10.5334/hpb.11.s1

- S File 2. Stress diary. DOI: https://doi.org/10.5334/ hpb.11.s2

- S File 3. Questionnaire. DOI: https://doi. org/10.5334/hpb.11.s3

- S File 4. Questionnaire 2. DOI: https://doi. org/10.5334/hpb.11.s4

- S File 5. Intervention timetable. DOI: https://doi. org/10.5334/hpb.11.s5

- S File 6. Ethical approval. DOI: https://doi. org/10.5334/hpb.11.s6

- S File 7. Datasheet. DOI: https://doi.org/10.5334/ hpb.11.s7

- S File 8. Syntax. DOI: https://doi.org/10.5334/ hpb.11.s8

- S File 9. Output. DOI: https://doi.org/10.5334/ hpb.11.s9

\section{Peer Review Comments}

Health Psychology Bulletin has blind peer review, which is unblinded upon article acceptance. The editorial history of this article can be downloaded here: 
- PR File 1. Editorial history. DOI: https://doi. org/10.5334/hpb.11.pr1

\section{Ethics and Consent}

This study has been approved by the local ethics committee of the faculty of Human Sciences at the University of Bern, Switzerland see Ethical_Approval in the Supplemental Material available online). All procedures performed in this study were in accordance with the ethical standards of the institutional and/or national research committee and with the 1964 Helsinki declaration and its later amendments. We obtained written, informed consent from the parents and the students prior to their inclusion in the study.

\section{Competing Interests}

The authors have no competing interests to declare.

\section{References}

Barak, A., Klein, B., \& Proudfoot, J. G. (2009). Defining internet-supported therapeutic interventions. Annals of Behavioral Medicine, 38, 4-17. DOI: https://doi.org/10.1007/s12160-009-9130-7

Blaser, M., \& Amstad, F. (2016). Psychische Gesundheit über die Lebensspanne [Psychological Health Across the Lifespan]. Bern: Gesundheitsförderung Schweiz.

Cohen, S., Janicki-Deverts, D., \& Miller, G. E. (2007). Psychological stress and disease. Jama, 298, 1685-1687. DOI: https://doi.org/10.1001/ jama.298.14.1685

Currie, C., Hurrelmann, K., Settertobulte, W., Smith, R., \& Todd, J. (2000). Health and health behaviour among young people. Issue 1 of the WHO Policy Series: Health policy for children and adolescents. Copenhagen: World Health Organization.

de Anda, D. (1998). The evaluation of a stress management program for middle school adolescents. Child and Adolescent Social Work Journal, 15, 73-85. DOI: https://doi.org/10.1023/A:1022297521709

de Anda, D., Baroni, S., Boskin, L., Buchwald, L., Morgan, J., Ow, J., Weiss, R., et al. (2000). Stress, stressors and coping strategies among high school students. Children and Youth Services Review, 22, 441-463. DOI: https://doi.org/10.1016/ S0190-7409(00)00096-7

Donaldson, D., Prinstein, M., Danovsky, M., \& Spirito, A. (2000). Patterns of children's coping with life stress: Implications for clinicians. American Journal of Orthopsychiatry, 70, 351-359. DOI: https://doi. org/10.1037/h0087689

Dube, S. R., Fairweather, D., Pearson, W. S., Felitti, V. J., Anda, R. F., \& Croft, J. B. (2009). Cumulative childhood stress and autoimmune diseases in adults. Psychosomatic Medicine, 71, 243-250. DOI: https://doi.org/10.1097/PSY.0b013e318190788

Eschenbeck, H., Kohlmann, C. W., Lohaus, A., \& Klein-Heßling, J. (2006). Die Diagnostik von Stressbewältigung mit dem "Fragebogen zur Erhebung von Stress und Stressbewältigung im Kindes-und
Jugendalter" (SSKJ 3-8) [The assessment of coping with the "Questionnaire for the Measurement Stress and Coping in Children and Adolescents" (SSKJ 3-8)]. Diagnostica, 52, 131-142. DOI: https://doi. org/10.1026/0012-1924.52.3.131

Faul, F., Erdfelder, E., Lang, A. G., \& Buchner, A. (2007). G*Power 3: A flexible statistical power analysis program for the social, behavioural, and biomedical sciences. Behavior Research Methods, 39, 175-191. DOI: https://doi.org/10.3758/BF03193146

Grant, K. E., Compas, B. E., Stuhlmacher, A. F., Thurm, A. E., McMahon, S. D., \& Halpert, J. A. (2003). Stressors and child and adolescent psychopathology: Moving from markers to mechanisms of risk. Psychological Bulletin, 129, 447-466. DOI: https:// doi.org/10.1037/0033-2909.129.3.447

Hampel, P., Meier, M., \& Kümmel, U. (2008). Schoolbased stress management training for adolescents: Longitudinal results from an experimental study. Journal of Youth and Adolescence, 37, 1009-1024. DOI: https://doi.org/10.1007/s10964-007-9204-4

Hampel, P., \& Petermann, F. (2003). Anti-Stress-Training für Kinder [Anti-Stress Training for Children]. Weinheim, Germany: Beltz.

Hampel, P., \& Petermann, F. (2016). Stressverarbeitungsfragebogen von Janke und Erdmann angepasst für Kinder und Jugendliche (SVF-KJ) [The German Coping Questionnaire by Janke and Erdmann Adapted for Children and Adolescents]. Göttingen, Germany: Hogrefe.

Inchley, J., Currie, C., Young, T., Samdal, O., Torsheim, T., Auguston, L., Barnekow, V., et al. (2016). Growing up unequal: Gender and socioeconomic differences in young people's health and well-being. Health Behaviour in School-aged Children (HBSC) Study: International Report from the 2013/14 Survey. Copenhagen: World Health Organization.

Klein-Heßling, J., \& Lohaus, A. (2000). Streßpräventionstraining für Kinder im Grundschulalter [Stress Prevention Training for Primary School Kids]. Göttingen, Germany: Hogrefe.

Lazarus, R. S., \& Folkman, S. (1984). Stress, appraisal, and coping. New York: Springer-Verlag.

Lohaus, A. (2010). Stress prevention in adolescence: Evaluation of a multimodal training approach. Journal of Public Health, 19, 385-388. DOI: https:// doi.org/10.1007/s10389-010-0391-1

Lohaus, A., Eschenbeck, H., Kohlmann, C. W., \& Klein-Heßling, J. (2006). Fragebogen zur Erhebung von Stress und Stressbewältigung im Kindes- und Jugendalter (SSKJ 3-8) [The German Stress and Coping Questionnaire for Children and Adolescents]. Göttingen, Germany: Hogrefe.

Lohaus, A., Fridrici, M., \& Maass, A. (2009). Stressprävention im Jugendalter: Effekte eines Trainingsprogramms mit Internetbegleitung [Stress prevention in adolescence: Effects of a training program with an accompanying internet platform]. Zeitschrift für Gesundheitspsychologie, 17, 13-21. DOI: https://doi. org/10.1026/0943-8149.17.1.13 
Lohaus, A., \& Nussbeck, F. W. (2016). Fragebogen zu Ressourcen im Kindes- und Jugendalter (FRKJ 8-16) [Resource Questionnaire for Children and Adolescents]. Göttingen, Germany: Hogrefe.

Matthews, K. A. (2005). Psychological perspectives on the development of coronary heart disease. American Psychologist, 60, 783-796. DOI: https:// doi.org/10.1037/0003-066X.60.8.783

McCallie, M. S., Blum, C. M., \& Hood, C. J. (2006). Progressive muscle relaxation. Journal of Human Behavior in the Social Environment, 13, 51-66. DOI: https://doi.org/10.1300/J137v13n03_04

McNamara, S. (2000). Stress in young people. What's new and what can we do? London: Continuum.

Meichenbaum, D. (1985). Stress inoculation training. New York: Pergamon Press.

Misra, R., \& McKean, M. (2000). College students' academic stress and its relation to their anxiety, time management, and leisure satisfaction. American Journal of Health Studies, 16, 41-51.

Power, T. G. (2004). Stress and coping in childhood: The parents' role. Parenting: Science and Practice,
4, 271-317. DOI: https://doi.org/10.1207/ s15327922par0404_1

Richards, D., \& Richardson, T. (2012). Computer-based psychological treatments for depression: A systematic review and meta-analysis. Clinical Psychology Review, 32, 329-342. DOI: https://doi. org/10.1016/j.cpr.2012.02.004

Seiffge-Krenke, I. (2000). Causal links between stressful events, coping style, and adolescent symptomatology. Journal of Adolescence, 23, 675-691. DOI: https://doi.org/10.1006/jado.2000.0352

Taylor, C. B., Jobson, K. O., Winzelberg, A., \& Abascal, L. (2002). The use of the Internet to provide evidence-based integrated treatment programs for mental health. Psychiatric Annals, 32, 671-677. DOI: https://doi.org/10.3928/0048-5713-20021101-06

Vandewater, E. A., Rideout, V. J., Wartella, E. A., Huang, X., Lee, J. H., \& Shim, M. S. (2007). Digital childhood: Electronic media and technology use among infants, toddlers, and preschoolers. Pediatrics, 119, 1006-1015. DOI: https://doi. org/10.1542/peds.2006-1804

How to cite this article: Englert, C., Bechler, A., Singh, S., and Bertrams, A. (2018). Testing the Effectiveness of a Short-Term Stress Prevention Programme in Primary School Students. Health Psychology Bulletin, 2(1), 1-9, DOl: https://doi.org/10.5334/ hpb.11

Submitted: 10 November 2017 Accepted: 22 June $2018 \quad$ Published: 13 July 2018

Copyright: $\odot 2018$ The Author(s). This is an open-access article distributed under the terms of the Creative Commons Attribution 4.0 International License (CC-BY 4.0), which permits unrestricted use, distribution, and reproduction in any medium, provided the original author and source are credited. See http://creativecommons.org/licenses/by/4.0/. 